La revue La revue pour l'histoire du CNRS

POUR L'HISTOIRE DU CNRS $\quad 8$ | 2003

Aux origines de l'Homme

Bizarre Big Bang. L'épopée de la physique

Philippe Miné. Belin - Pour la science, Paris, 2001

Michel Paty

(2) OpenEdition

Journals

Édition électronique

URL : https://journals.openedition.org/histoire-cnrs/417

DOI : 10.4000/histoire-cnrs.417

ISSN : 1955-2408

Éditeur

CNRS Éditions

Édition imprimée

Date de publication : 5 mai 2003

ISBN : 978-2-271-06068-6

ISSN : $1298-9800$

Référence électronique

Michel Paty, «Bizarre Big Bang. L'épopée de la physique », La revue pour l'histoire du CNRS [En ligne], 8 | 2003, mis en ligne le 07 mars 2006, consulté le 20 mai 2021. URL : http://journals.openedition.org/ histoire-cnrs/417 ; DOI : https://doi.org/10.4000/histoire-cnrs.417

Ce document a été généré automatiquement le 20 mai 2021

Comité pour l'histoire du CNRS 


\section{Bizarre Big Bang. L'épopée de la physique}

Philippe Miné. Belin - Pour la science, Paris, 2001

\section{Michel Paty}

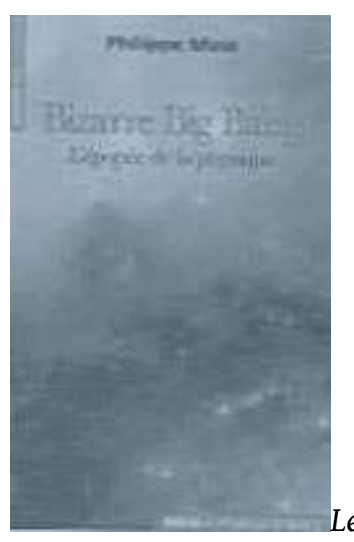

e Big Bang ou le mythe raisonné et magnifié par la science. Le Big Bang est, comme chacun sait, la théorie actuelle la plus sérieuse du monde, si l'on ose dire, de la science cosmologique, malgré son nom qui, lui, ne fait pas très sérieux, il faut bien en convenir. Et c'est sans doute pour cette double raison contradictoire qu'il fait florès ces temps-ci, banalisé dans le langage courant en métaphore pour tous services. Il est vrai qu'aux «big bang des fonds de pension» et autres «big bang au parti socialiste ", justement épinglés par l'auteur, je préfère, pour ma part, celui, avec un B majuscule, dont le jeune fils de Trevor expliquait la signification à son père, parti à sa recherche et qui venait de le retrouver après plusieurs péripéties, dans le film ParisTexas de Wim Wenders. C'était le signe de la nouvelle mythologie de l'Univers que les enfants peuvent capter à la télévision dans les villes anonymes.

1 Le mot semble bien porter avec lui le mythe, qui lui fut à l'origine collé par dérision par un qui n'aimait pas l'idée, Fred Hoyle, comme le rappelle l'auteur. il décortique de façon gourmande, comme le yin et le yang. Mais voilà que la dérision s'est retournée, la controverse effacée sous le poids des succès, le consensus largement établi, et que l'hypothèse en question est désormais considérée, malgré son ambition, ou plutôt, dans 
son ambition même, comme l'une des plus puissantes qu'ait jamais produites la physique. Une physique étendant, il est vrai, ses objets, divers mais saisis dans l'unité celle de la matière et de ses représentations-, à cet objet unique et singulier qu'est l'Univers lui-même dans sa totalité, pris désormais comme objet de science, au sens le plus positif du mot. Et, avec lui, la cosmologie, autrefois objet des mythes, du Timée de Platon à ceux des anciens Égyptiens ou des Mayas, ou de la pensée métaphysique comme les sphères cristallines emboîtées de la cosmologie géocentrique d'Aristote et Ptolémée qui perdura jusqu'à Copernic, cette cosmologie devait connaître la plus grande des transformations. Comme l'a analysé, dans ses beaux travaux pionniers d'épistémologie de la cosmologie, le philosophe récemment disparu Jacques MerleauPonty (1917-2002) (dont on regrette de ne pas trouver mention dans ce livre), l'avènement de la physique moderne post-copernicienne s'est accompagné, du XVII ${ }^{e}$ siècle au début du $\mathrm{XX}^{\mathrm{e}}$, d'une relégation (par ailleurs féconde) de l'idée cosmologique dans le sens d'une validité universelle, en tout lieu du cosmos, des lois de la nature, l'étude précise, locale et analytique des objets et des phénomènes excluant d'y comprendre leur totalité et l'Univers lui-même.

2 La "révolution cosmologique du XXe siècle », survenue dans les années 1920, rétablit de manière spectaculaire et, semble-t-il, irréversible, l'Univers comme objet de pensée, et de pensée scientifique. Elle le fit du côté théorique par la relativité générale d'Einstein, qui posait le problème de la nature de l'espace aux confins de la matière, et donc de l'Univers comme un tout ; et du côté phénoménal par l'observation de la récession des galaxies d'où l'on inféra l'expansion de l'Univers. Un second moment dort de l'approche scientifique de la cosmologie fut, dès le milieu des années 1960, la conjugaison d'observations sur l'abondance des éléments chimiques légers et l'observation du rayonnement électromagnétique radio (centimétrique) isotrope fossile, comme témoin des premières périodes où l'Univers était très chaud, dense et ramassé sur lui-même. Depuis lors, ces idées n'ont fait que se préciser, et la structuration présente et future de l'Univers est conçue comme ayant été en germe dans ses premiers instants, où son état matériel était soumis aux lois de la physique quantique. La physique du cosmos et celle des particules subatomiques connaissent ainsi de nos jours un rapprochement, et jusqu'à un certain point une identification, qui aurait été impensable naguère. Voici donc quelles sont les implications de cette étrange théorie du Big Bang, par-delà la bizarrerie apparente des termes qui la désignent.

3 C'est le grand mérite du livre de Philippe Miné, chercheur en physique des particules subnucléaires, de présenter d'une manière lisible par le non-spécialiste, et agréable à lire, les éléments de connaissance les plus significatifs de cette aventure (qu'on n'hésite pas à dire, sans exagération, splendide) de la pensée scientifique. On admirera, en particulier, la rigueur et la clarté de ses descriptions et explications, qui ne cèdent jamais à la tentation des métaphores faciles et trompeuses, comme c'est trop souvent le cas pour ce domaine. On dispose avec cet ouvrage d'un état de l'art, informé de première main, et très complet, de la "physique des objets extrêmes", particules subatomiques et cosmos, de leurs tenants et aboutissants (avec des retours dans l'histoire des idées à l'origine des concepts, comme celui d'espace non euclidien, par exemple), du neutrino au trou noir, du Big Bang passé au (possible) Big Crunch futur. C'est un exposé raisonné, par lequel l'auteur s'efforce de répondre le plus honnêtement et raisonnablement du monde à la demande du lecteur, dont la curiosité est légitimement éveillée sur ces questions. Les livres de ce genre sont un complément indispensable (et démystificateur) aux avancées de la recherche scientifique, qu'ils 
mettent de façon simple à la portée d'un public assez large, en lui faisant voir l'état des connaissances actuelles, et la dynamique qui les pousse en avant par les questions mêmes qu'elles soulèvent. Ils sont aussi, comme l'auteur en est bien conscient, une exigence du partage démocratique nécessaire des connaissances.

INDEX

Mots-clés : physique, Big Bang, Univers, épopée, révolution cosmologique

\section{AUTEUR}

MICHEL PATY

Directeur de recherche au CNRS 